\title{
Lattice QCD study of static quark and antiquark correlations via entanglement entropies
}

\author{
Toru T. Takahashi ${ }^{1}$ and Yoshiko Kanada-En'yo ${ }^{2}$ \\ ${ }^{1}$ National Institute of Technology, Gunma college, Maebashi, Gunma 371-8530, Japan \\ ${ }^{2}$ Department of Physics, Kyoto University, Sakyo, Kyoto 606-8502, Japan
}

(Received 17 October 2019; published 4 December 2019)

\begin{abstract}
We study the color correlation between the static quark and antiquark $(q \bar{q})$ in the confined phase via reduced density matrices $\rho$ defined in color space. We adopt the standard Wilson gauge action and perform quenched calculations with the Coulomb gauge condition for reduced density matrices. The spatial volumes are $L^{3}=8^{3}, 16^{3}, 32^{3}$, and $48^{3}$, with the gauge couplings $\beta=5.7,5.8$, and 6.0. Each element of the reduced density matrix in the subspace of quarks' color degrees of freedom of the $q \bar{q}$ pair is calculated from staples defined by link variables. As a result, we find that $\rho$ is well written by a linear combination of the strongly correlated $q \bar{q}$ pair state with the color-singlet component and the uncorrelated $q \bar{q}$ pair state with random color configurations. We compute the Renyi entropies $S^{\text {Renyi }}$ from $\rho$ to investigate the $q \bar{q}$ distance dependence of the color correlation of the $q \bar{q}$ pair and find that the color correlation is quenched as the distance increases.
\end{abstract}

DOI: $10.1103 /$ PhysRevD.100.114502

\section{INTRODUCTION}

Color confinement is one of the nonperturbative features of QCD, the fundamental theory of the strong interaction. The static interquark potential ( $q \bar{q}$ potential) in the confinement phase exhibits a linearly rising potential in the largeseparation limit giving the diverging energy, and quarks cannot be isolated. Such confining features have been studied and confirmed in several approaches [1].

The color confinement may be illustrated by the flux tube formation between a quark and antiquark. A color flux tube which has a constant energy per length is formed between a (color-singlet) $q \bar{q}$ pair, and this tube gives the linearly rising $q \bar{q}$ potential $[2,3]$. Note that QCD is nonAbelian gauge theory, and hence such gluon fluxes have colors. In other words, the color charge first associated with a color-singlet $q \bar{q}$ pair flows into the interquark flux tube as the $q \bar{q}$ separation is enlarged, keeping the total system color singlet [4,5]. If the color charge of the $q \bar{q}$ part and that of the gluon part are separately considered, this color transfer can be regarded as a color charge leak from the $q \bar{q}$ part to the gluon part in association with the screening effect. This color leak should depend on the $q \bar{q}$ distance and would be observed as the distance dependence of the color correlation between the quark and antiquark.

Published by the American Physical Society under the terms of the Creative Commons Attribution 4.0 International license. Further distribution of this work must maintain attribution to the author(s) and the published article's title, journal citation, and DOI. Funded by SCOAP .
Such color correlation of the $q \bar{q}$ pair may be detected by entanglement entropy (EE) defined by the reduced density matrix. EE quantifies an entanglement between degrees of freedom (d.o.f.) in purely quantum systems and has been utilized in variety of physical systems [6-17]. If the $q \bar{q}$ pair's correlation is strong, the $q \bar{q}$ part is well decoupled from the gluon part, and there is no entanglement between the $q \bar{q}$ and gluon parts. In other words, the color leak from the $q \bar{q}$ part can be measured by EE. In this paper, we define the reduced density matrix $\rho$ for a static $q \bar{q}$ pair in terms of color d.o.f. The density matrix is reduced into subspace of $q \bar{q}$ color configurations by integrating out the gluons' d.o.f., which is simply done by averaging the density matrix components over gauge configurations, and computing entanglement entropy $S$ with the reduced density matrix. Constructing a simple ansatz for the reduced density matrix $\rho$, we investigate the dependence of $S$ on the interquark distance $R$.

In Sec. II, we give the formalism to compute the reduced density matrix $\rho$ of $q \bar{q}$ system and the entanglement entropy $S$ of it. The details of numerical calculations and ansatz for $\rho$ are also shown in Sec. II. Results are presented in Sec. III. Section IV is devoted to the summary and concluding remarks.

\section{FORMALISM}

\section{A. Reduced two-body density matrix and $q \bar{q}$ correlation}

The entanglement between two subsystems $A$ and $B$ can be quantified with EE. From the density matrix $\rho_{A B}$ for a whole system $A+B$, the reduced density matrix $\rho_{A}$ is 
obtained as $\rho_{A}=\operatorname{Tr}_{B}\left(\rho_{A B}\right)$. Here, $\operatorname{Tr}_{B}$ is taken over the d.o.f. of the subsystem $B$. The entanglement entropy $S_{A}^{\mathrm{EE}}$ of the subsystem $A$ is then defined as $S_{A}^{\mathrm{EE}}=-\operatorname{Tr}_{A}\left(\rho_{A} \log \rho_{A}\right)$ in the functional form of the von Neumann entropy. The density matrix $\rho_{A}$ defined for the reduced space (the subsystem A) can give a nonzero value of EE because a part of information is lost from the $\rho_{A B}$ for the full space by tracing out d.o.f. of the subsystem $B$. The EE is zero only in the case of $\rho_{A}^{2}=\rho_{A}$ when the subsystems $A$ and $B$ are completely decoupled from each other (not entangled).

Since our interest is being focused on the static $q \bar{q}$ pair's color correlations, we divide the whole color-singlet system into two (possibly colored) subsystems, static (anti)quarks $(Q)$, and "others" $(G)$ and consider color DOF of the subsystems $(Q=A$ and $G=B)$. Other DOF contain all the gluon's DOF including the vacuum polarization by the sea quark's loop.

In the actual calculations, we compute the reduced twobody density matrix $\rho$ in the subsystem $Q$ by taking into account the static quark's color configuration only. The thus defined density matrix is nothing but the reduced density matrix $\rho_{Q}$ that is obtained by integrating out the other DOF $G$ in the full density matrix $\rho_{Q G} ; \rho_{Q}=\operatorname{Tr}_{G}\left(\rho_{Q G}\right)$.

The reduced two-body density operator $\hat{\rho}(R)$ in a $q \bar{q}$ system with the interquark distance $R$ is defined as

$$
\hat{\rho}(R)=|\bar{q}(0) q(R)\rangle\langle\bar{q}(0) q(R)| .
$$

Here, $|\bar{q}(0) q(R)\rangle$ represents a quantum state in which the antiquark is located at the origin and the other quark lies at $x=R$. The reduced density matrix components $\rho(R)_{i j, k l}$, where $i(j)$ are quark's (antiquark's) color indices, are expressed as

$$
\rho(R)_{i j, k l}=\left\langle q_{i}(0) \bar{q}_{j}(R)|\hat{\rho}(R)| q_{k}(0) \bar{q}_{l}(R)\right\rangle .
$$

$\rho(R)$ is a $m \times m$ square matrix with the dimension $m=N_{c}^{2}$. Note again that $\rho$ is defined using only the quark's DOF and the gluon's wave function is not considered, and then the thus-defined $\rho$ can be regarded as a reduced density matrix in which the gluon's DOF are integrated out.

The von Neumann (VN) entanglement entropy $S^{\mathrm{VN}}(R)$ for a $q \bar{q}$ pair at a distance of $R$ can be computed with the reduced density matrix $\rho(R)$ as

$$
S^{\mathrm{VN}}(R) \equiv-\operatorname{Tr} \rho(R) \log \rho(R)=-\sum_{i j}[\rho(R) \log \rho(R)]_{i j},
$$

which can be regarded as an entanglement entropy representing the correlation between static quark pair (subsystem $Q$ ) and other DOF (subsystem $G$ ).

In the actual computation of $S^{\mathrm{VN}}$, one needs to diagonalize $\rho$ or approximate the logarithmic function. To avoid such a numerically demanding processes, we adopt Renyi entropy [18] for EE for detailed analysis. Renyi entanglement entropy $S^{\text {Renyi- } \alpha}$ of order $\alpha(\alpha>0, \alpha \neq 1)$ is given as

$$
S^{\text {Renyi- }-\alpha}=\frac{1}{1-\alpha} \log \operatorname{Tr}\left(\rho^{\alpha}\right),
$$

with a reduced density matrix $\rho$. Note that in the limit when $\alpha \rightarrow 1$, it goes to von Neumann entropy as $S^{\text {Renyi- } \alpha} \rightarrow S^{\mathrm{VN}}$. Renyi entanglement entropy is a kind of generalized entropies that quantify uncertainty or randomness and is used to measure entanglement in quantum information theory. Since entanglement entropy is invariant under unitary transformations, it enables representation-independent analysis. We use the second-order Renyi entanglement entropy by taking $\alpha=2$, which is simply given by the squared $\rho(R)$ as

$$
S^{\text {Renyi-2 }}=-\log \operatorname{Tr}\left(\rho^{2}\right) .
$$

We comment here on the relationship between $q \bar{q}$ correlation and the entanglement entropy. Our main interest is the $q \bar{q}$ pair's color correlation defined in the subsystem $Q$. The whole pure state in the $Q+G$ system can be written as

$$
\sum_{\alpha}|\alpha\rangle_{Q} \otimes|\alpha\rangle_{G}
$$

Here, $\alpha$ denotes all the possible color states of the $q \bar{q}$ pair, and the total system is kept in a color-singlet state. When the quark and antiquark's colors are strongly correlated forming a color-singlet combination $|\mathbf{1}\rangle_{Q}$ with no color charge leak from $Q$ to $G$, the subsystems $Q$ and $G$ are well decoupled in the color space, and therefore the whole state can be expressed in a simple product of $Q$ and $G$ parts as

$$
\sum_{\alpha=\mathbf{1}}|\alpha\rangle_{Q} \otimes|\alpha\rangle_{G}=|\mathbf{1}\rangle_{Q} \otimes|\mathbf{1}\rangle_{G}
$$

In this strongly correlated case, the entanglement entropy $S^{\mathrm{EE}}$ goes to zero, since two subsystems $Q$ and $G$ decouple and the entanglement between subsystems $Q$ and $G$ vanishes.

On the other hand, when a $q \bar{q}$ pair's color charge leaks into in between gluons and the color correlation between them decreases, the whole state cannot be written in a separable form, and $S$ would take a positive finite value as $S>0$.

\section{B. Ansatz for reduced density matrix $\rho_{i j, k l}(R)$}

Let us consider a possible functional form of the reduced density matrix $\rho_{i j, k l}(R)$ based on the simple ansatz that the contamination mixed to the correlated color-singlet component is the random color component without any color correlation between the quark and antiquark of the $q \bar{q}$ pair. We first define the density operator $\hat{\rho}_{s, s}$ for the quark and antiquark in a color-singlet state $|\boldsymbol{s}\rangle=\sum_{i}^{N_{c}}\left|\bar{q}_{i} q_{i}\right\rangle$ in the Coulomb gauge as

$$
\hat{\rho}_{s, s}=|s\rangle\langle s| .
$$


In color $\mathrm{SU}\left(N_{c}\right) \mathrm{QCD}$, the density operator $\hat{\rho}_{\boldsymbol{a}_{\boldsymbol{i}}, \boldsymbol{a}_{i}}(i=$ $\left.1,2, \ldots, N_{c}^{2}-1\right)$ for $q \bar{q}$ in an adjoint state $\left|\boldsymbol{a}_{i}\right\rangle(i=$ $\left.1,2, \ldots, N_{c}^{2}-1\right)$ is expressed as

$$
\hat{\rho}_{\boldsymbol{a}_{i}, \boldsymbol{a}_{i}}=\left|\boldsymbol{a}_{i}\right\rangle\left\langle\boldsymbol{a}_{i}\right|\left(i=1,2, \ldots, N_{c}^{2}-1\right) .
$$

In the limit $R \rightarrow 0$, the quark and antiquark are considered to form a color-singlet state $(|\boldsymbol{s}\rangle)$ corresponding to the strong correlation limit, and its density operator will be written as

$$
\hat{\rho}^{0}=\hat{\rho}_{s, s}=\operatorname{diag}(1,0, \ldots, 0)_{\alpha-\text { rep. }}
$$

Here, " $\alpha$ - rep." means that the matrix is expressed in terms of $q \bar{q}$ 's color representation with the vector set of $\left\{\boldsymbol{s}, \boldsymbol{a}_{1}, \ldots \boldsymbol{a}_{8}\right\}$. As $R$ increases, it is expected that adjoint components mix into the singlet component due to the QCD interaction. We assume that contamination mixed into the pure singlet (correlated) state is the uncorrelated state with random color configurations in which $N_{c}^{2}$ components mix with equal weights. The density operator for such the random state is given as

$$
\begin{aligned}
\hat{\rho}^{\text {rand }} & =\frac{1}{N_{c}^{2}} \hat{\rho}_{\boldsymbol{s}, s}+\frac{1}{N_{c}^{2}} \hat{\rho}_{\boldsymbol{a}_{1}, \boldsymbol{a}_{1}}+\frac{1}{N_{c}^{2}} \hat{\rho}_{\boldsymbol{a}_{2}, \boldsymbol{a}_{2}}+\cdots \\
& =\frac{1}{N_{c}^{2}} \hat{I}=\frac{1}{N_{c}^{2}} \operatorname{diag}(1,1, \ldots, 1)_{\alpha-\text { rep. }}
\end{aligned}
$$

Letting the fraction of the original (maximally correlated) singlet state be $F(R)$ and that of the mixing (random) components be $(1-F(R))$, the density operator in this ansatz is written as

$$
\hat{\rho}_{\text {ansatz }}(R)=F(R) \hat{\rho}^{0}+(1-F(R)) \hat{\rho}^{\text {rand }} .
$$

The matrix elements of $\hat{\rho}_{\text {ansatz }}(R)$ in the $\alpha$-representation are explicitly written as

$$
\begin{aligned}
\hat{\rho}_{\text {ansatz }}(R) & =F(R) \hat{\rho}^{0}+(1-F(R)) \hat{\rho}^{\text {rand }} \\
& =\operatorname{diag}\left(F(R)+\frac{1}{N_{c}^{2}}(1-F(R)), \frac{1}{N_{c}^{2}}(1-F(R)), \ldots, \frac{1}{N_{c}^{2}}(1-F(R))\right)_{\alpha-\text { rep. }} \\
& =\left(\begin{array}{cccc}
F(R)+\frac{1}{N_{c}^{2}}(1-F(R)) & 0 & \cdots & 0 \\
0 & \frac{1}{N_{c}^{2}}(1-F(R)) & \vdots \\
\vdots & & \ddots & \vdots \\
\vdots & \ldots & & 0 \\
0 & & & \frac{1}{N_{c}^{2}}(1-F(R))
\end{array}\right)_{\alpha-\text { rep. }}
\end{aligned}
$$

When $N_{c}=3$,

$\left\{\begin{array}{l}\rho(R)_{\mathbf{8}_{1}, \mathbf{8}_{1}}=\rho(R)_{\mathbf{8}_{2}, \mathbf{8}_{2}}=\cdots=\rho(R)_{\mathbf{8}_{8}, \mathbf{8}_{8}} \equiv \rho(R)_{\mathbf{8}, \mathbf{8}} \\ \rho(R)_{\alpha, \beta}=0 \quad(\text { for } \alpha \neq \beta)\end{array}\right.$

would be satisfied at any $R$ in this ansatz. The first relation should be satisfied due to the color SU(3) symmetry. The second, which means that the off-diagonal components are all zero, comes from the ansatz of the random state. The normalization condition $\operatorname{Tr} \rho=1$ is trivially satisfied in this ansatz as

$$
\rho(R)_{\mathbf{1}, \mathbf{1}}+\left(N_{c}^{2}-1\right) \rho(R)_{\mathbf{8 , 8}}=1 .
$$

In the strong correlation limit when the $q \bar{q}$ pair's color forms $|\mathbf{1}\rangle, F(R)=1$. On the other hand, in the random limit when quarks' colors are screened, $F(R)=0$.

\section{Lattice QCD formalism}

Let the site on the lattice be $\boldsymbol{r}=(x, y, z, t)=x \boldsymbol{e}_{x}+$ $y \boldsymbol{e}_{y}+z \boldsymbol{e}_{z}+t \boldsymbol{e}_{t}$ and $\mu$ direction $(\mu=x, y, z, t)$ link variables be $U_{\mu}(\boldsymbol{r})$. With a lower staple $S^{L}(R, T)$ representing $q \bar{q}$ pair creation and propagation and an upper staple $S^{U}(R, T)$ for $q \bar{q}$ pair annihilation that are defined as

$$
\begin{aligned}
S_{i j}^{L}(R, T) \equiv & \left(\prod_{t=-1}^{-T} U_{t}^{\dagger}\left(t \boldsymbol{e}_{t}\right) \prod_{x=0}^{R-1} U_{x}\left(x \boldsymbol{e}_{x}-T \boldsymbol{e}_{t}\right)\right. \\
& \left.\times \prod_{t=-T}^{-1} U_{t}\left(R \boldsymbol{e}_{x}+t \boldsymbol{e}_{t}\right)\right)_{i j} \\
S_{i j}^{U}(R, T) \equiv & \left(\prod_{t=0}^{T-1} U_{t}\left(t \boldsymbol{e}_{t}\right) \prod_{x=0}^{R-1} U_{x}\left(x \boldsymbol{e}_{x}+T \boldsymbol{e}_{t}\right)\right. \\
& \left.\times \prod_{t=T-1}^{0} U_{t}^{\dagger}\left(R \boldsymbol{e}_{x}+t \boldsymbol{e}_{t}\right)\right)_{i j}
\end{aligned}
$$


we define $L_{i j}(R, T)$ as

$$
L_{i j, k l}(R, T) \equiv S_{i j}^{U}(R, T) S_{k l}^{L \dagger}(R, T)
$$

When the Euclidean time separation $T$ is large enough and excited-state contributions can be ignored, $\left\langle L_{i j, k l}(R, T)\right\rangle$ is expressed as

$$
\begin{aligned}
& \left\langle L_{i j, k l}(R, T)\right\rangle \\
& =C\left\langle q(0) \bar{q}(R)\left|e^{-\hat{H} T}\right| q_{i}(0) \bar{q}_{j}(R)\right\rangle \\
& \quad \times\left\langle\bar{q}_{k}(0) q_{l}(R)\left|e^{-\hat{H} T}\right| q(0) \bar{q}(R)\right\rangle \\
& =C e^{-2 E_{0} T}\left\langle q(0) \bar{q}(R) \mid q_{i}(0) \bar{q}_{j}(R)\right\rangle\left\langle\bar{q}_{k}(0) q_{l}(R) \mid q(0) \bar{q}(R)\right\rangle \\
& =C e^{-2 E_{0} T} \rho(R)_{i j, k l},
\end{aligned}
$$

where $E_{0}$ is the ground-state energy. Normalizing $\langle L(R, T)\rangle$ so that $\operatorname{Tr}\langle L(R, T)\rangle=\sum_{i j}\left\langle L_{i j, i j}(R, T)\right\rangle=1$, we obtain $\rho(R)$, the trace of which is unity $(\operatorname{Tr} \rho(R)=1)$.

Once we obtain $\rho(R)$, Renyi entropy of order $\alpha$ as a function of $R$ is obtained as

$$
S^{\text {Renyi }-\alpha}(R)=\frac{1}{1-\alpha} \log \operatorname{Tr}\left(\rho(R)^{\alpha}\right)
$$

\section{Lattice QCD parameters}

We adopt the standard Wilson gauge action and perform quenched calculations for reduced density matrices of static quark and antiquark $(q \bar{q})$ systems. The gauge configurations are generated on the spatial volumes $L^{3}=8^{3}, 16^{3}$, $32^{3}$, and $48^{3}$, with the gauge couplings $\beta=5.7,5.8$, and 6.0. All the gauge configurations are gauge fixed with the Coulomb gauge condition. The parameters adopted in the present work are summarized in Table I.

TABLE I. Lattice QCD parameters. Coupling $\beta$, lattice spacing $a$, and spatial volume $L^{3}$ in the lattice unit and the physical unit.

\begin{tabular}{lccc}
\hline \hline$\beta$ & $a(\mathrm{fm})$ & $L^{3}$ & $L^{3}\left(\mathrm{fm}^{3}\right)$ \\
\hline 5.7 & 0.18 & $8^{3}$ & $1.44^{3}$ \\
5.7 & 0.18 & $16^{3}$ & $2.88^{3}$ \\
5.7 & 0.18 & $32^{3}$ & $5.75^{3}$ \\
5.7 & 0.18 & $48^{3}$ & $8.64^{3}$ \\
5.8 & 0.14 & $16^{3}$ & $2.24^{3}$ \\
5.8 & 0.14 & $32^{3}$ & $4.48^{3}$ \\
5.8 & 0.14 & $48^{3}$ & $6.72^{3}$ \\
6.0 & 0.10 & $16^{3}$ & $1.60^{3}$ \\
6.0 & 0.10 & $32^{3}$ & $3.20^{3}$ \\
6.0 & 0.10 & $48^{3}$ & $4.80^{3}$ \\
\hline \hline
\end{tabular}

\section{LATTICE QCD RESULTS}

\section{A. Ground-state dominance}

To confirm the ground-state dominance, we investigate the static quark and antiquark potential. In Figs. 1, 2, and 3, we show the effective energy plots for static $q \bar{q}$ systems with several interquark distances $R$ as a function of the Euclidean time separation $T$. For all the interquark distances $R$, effective energies show plateaus against $T$ at $T \geq 2$, and it is confirmed that ground-state saturation is ensured at $T \geq 2$. Hereafter, we adopt normalized reduced density matrix $\rho(R, 2)$ measured with $T=2$ for $\rho_{i j, k l}(R) ; \rho_{i j, k l}(R) \equiv \rho_{i j, k l}(R, 2) / \operatorname{Tr} \rho(R, 2)$.

\section{B. Reduced density matrix elements}

In this subsection, we take a detailed look at the reduced density matrix elements obtained with lattice QCD. To see the validity of the first condition in Eq. (16), we define the average

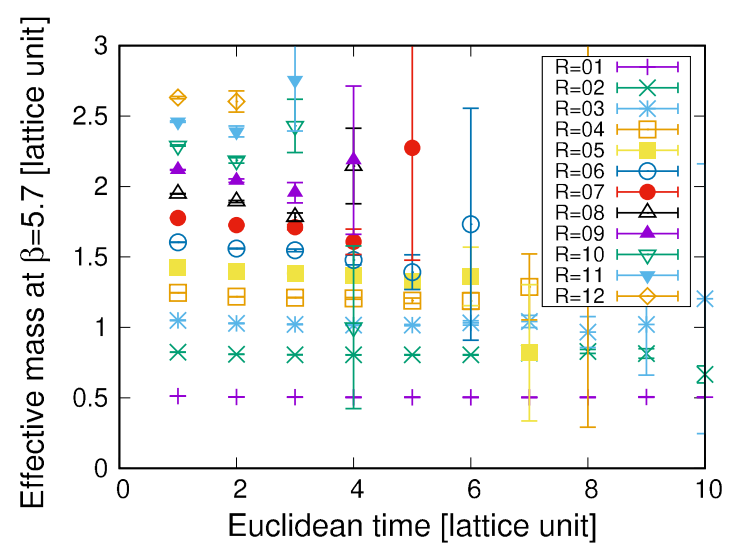

FIG. 1. Effective energy plot as a function of the Euclidean time separation at $\beta=5.7$. $R$ denotes the interquark distance in lattice unit.

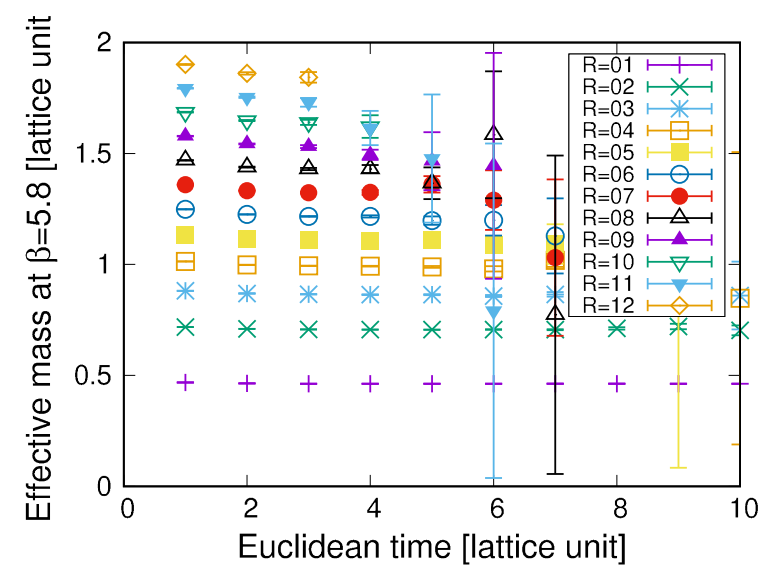

FIG. 2. Effective energy plot as a function of the Euclidean time separation at $\beta=5.8$. $R$ denotes the interquark distance in lattice units. 


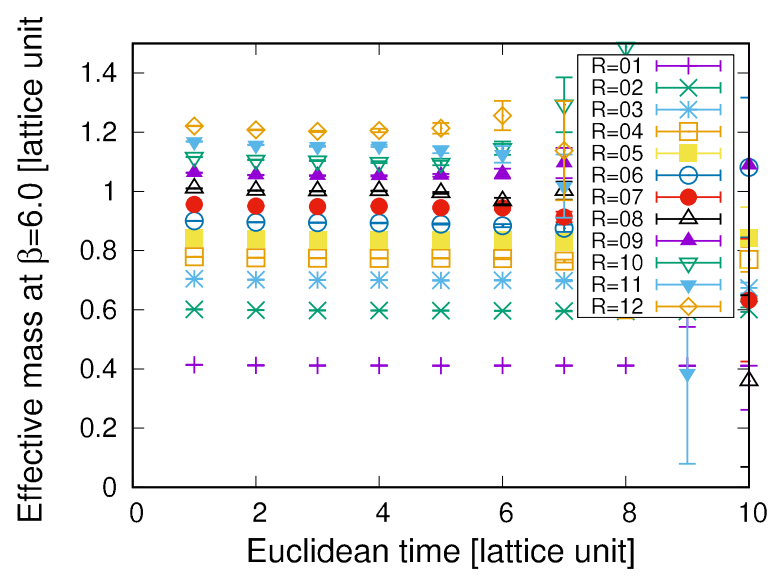

FIG. 3. Effective energy plot as a function of the Euclidean time separation at $\beta=6.0 . R$ denotes the interquark distance in lattice units.

$$
\rho(R)_{\mathbf{8}, \mathbf{8}}=\frac{1}{N_{c}^{2}-1} \sum_{i} \rho(R)_{\mathbf{8}_{i}, \mathbf{s}_{i}}
$$

and the deviation

$$
D_{i}(R)=\rho(R)_{\mathbf{8}_{i}, \boldsymbol{8}_{i}}-\rho(R)_{\mathbf{8}, \mathbf{8}}
$$

In Fig. $4, D_{i}(R)(1 \leq i \leq 8)$ are plotted as a function of the interquark distance. All the values are consistent with zero, and it is confirmed that the first condition is satisfied for all the $R$ and $i$ within statistical errors. Hereafter, the octet components of $\rho(R)$ are represented by the averaged value $\rho(R)_{\mathbf{8 , 8}}$.

The second condition in Eq. (16) is the assumption in the ansatz. To see to what extent this assumption is valid in the actual reduced density matrices, we define following two independent components:

$$
\begin{gathered}
\rho(R)_{\mathbf{1 , \mathbf { 8 } _ { 1 }}}=-\frac{1}{\sqrt{3}}\left(\rho(R)_{11,12}+\rho(R)_{22,12}+\rho(R)_{33,12}\right), \\
\rho(R)_{\mathbf{8}_{3}, \mathbf{8}_{4}}=\rho(R)_{21,13} .
\end{gathered}
$$

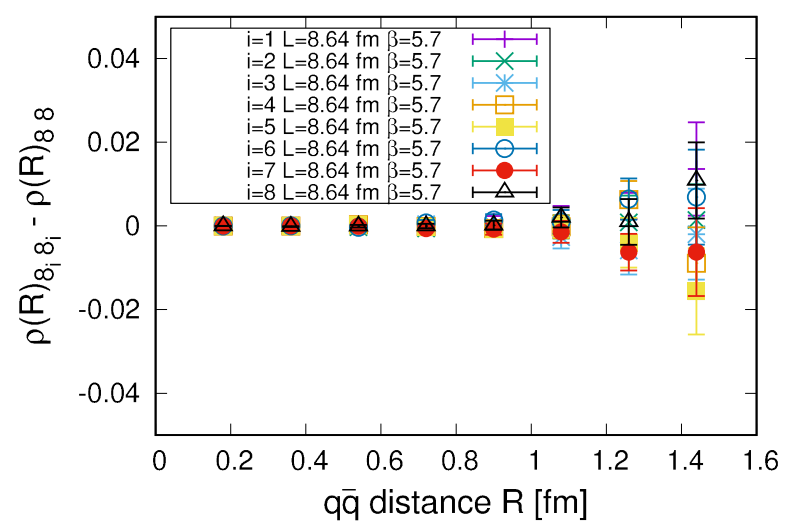

FIG. 4. The deviation of each component $\rho(R)_{\mathbf{8}_{i}, \mathbf{8}_{i}}$ from the averaged value $\rho(R)_{\mathbf{8 , 8}}=\frac{1}{N_{c}^{2}-1} \sum_{i} \rho(R)_{\mathbf{8}_{i}, \mathbf{8}_{i}}$ is plotted as a function of the interquark distance. They are evaluated at $\beta=5.7$ and $L=48$. All the values are consistent with zero within the errors.

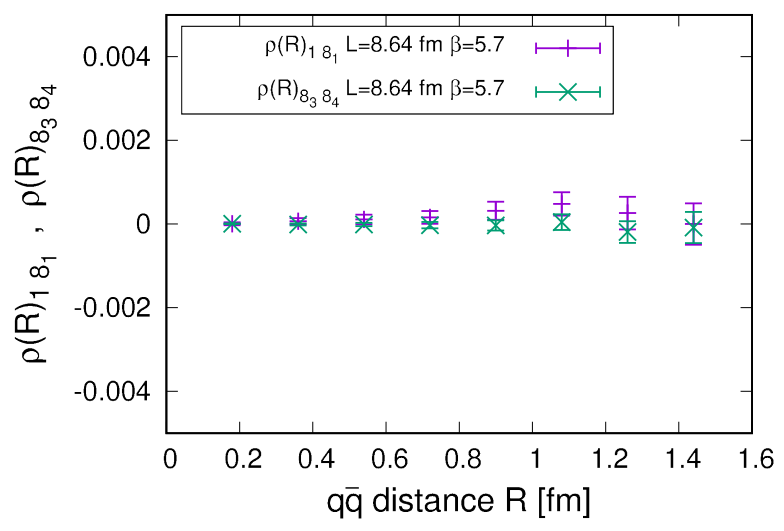

FIG. 5. To see the magnitudes of the off-diagonal components, two independent off-diagonal components $\rho(R)_{\mathbf{1}, \mathbf{8}_{1}}$ and $\rho(R)_{\mathbf{8}_{3}, \mathbf{8}_{4}}$ are plotted as a function of the interquark distance. They are consistent with zero, and we conjecture that the off-diagonal components of the reduced density matrix $\rho(R)$ are considerably small.

$\rho(R)_{1, \mathbf{8}_{1}}$ and $\rho(R)_{\mathbf{8}_{3}, \mathbf{8}_{4}}$ are plotted in Fig. 5. We find that they are consistent with zero, and we conjecture that the offdiagonal components of the reduced density matrix $\rho(R)$ are considerably small. From these analyses, we can conclude that the reduced density matrix $\rho(R)$ obtained with lattice calculations in the static $q \bar{q}$ system is expressed by the ansatz with high accuracy. Indeed, even when we replace the octet components and the off-diagonal components of $\rho(R)$ with the average $\rho(R)_{\mathbf{8}, \mathbf{8}}$ and with zero by hand, all the results remain almost unchanged.

\section{C. $\boldsymbol{R}$ dependence of $\boldsymbol{F}(\boldsymbol{R})$}

Taking into account the normalization condition

$$
\rho(R)_{\mathbf{1}, \mathbf{1}}+\left(N_{c}^{2}-1\right) \rho(R)_{\mathbf{8 , 8}}=1,
$$

the independent quantity at a given $R$ is only $\rho_{\mathbf{8 , 8}}$, and we can compute the fraction $F(R)$ of the remaining correlated $q \bar{q}$ component as

$$
F(R)=\rho(R)_{\mathbf{1 , 1}}-\rho(R)_{\mathbf{8}, \mathbf{8}}=1-N_{c}^{2} \rho(R)_{\mathbf{8}, \mathbf{8}} .
$$

When the $q \bar{q}$ system forms a random state with no color correlation between $q$ and $\bar{q}$, the calculated $\rho(R)$ equals $\hat{\rho}^{\text {rand }}$ and gives $F(R)=0$. In the upper panel in Fig. 6, $F(R)$ is plotted as a function of the interquark distance $R . F(R)$ linearly decreases at small $R$ and exponentially approaches zero at large $R$, which can be also seen in the lower panel [logarithmic plot of $F(R)$ ].

The exponential decay of the $q \bar{q}$ correlation indicates the color screening effects due to in-between gluons. We fit $F(R)$ with an exponential function as

$$
F(R)=A \exp (-B R)
$$

and extract the "screening mass" $B$. In Fig. 7, the fitted parameters $A$ and $B$ are plotted as functions of the spatial lattice size $L$. The plot includes all the data obtained at 

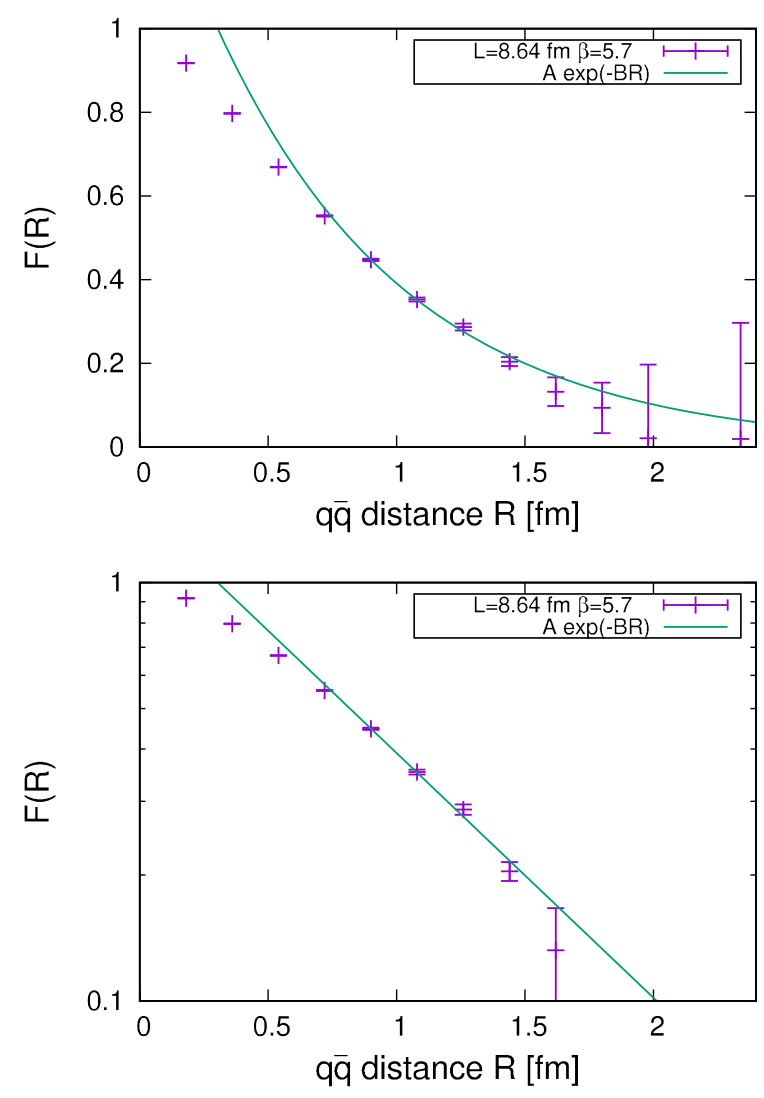

FIG. 6. $\quad F(R)$ is plotted as a function of the interquark distance $R$ in the upper panel. $F(R)$ monotonously decreases and approaches zero. The lower panel shows the log plot for $F(R)$. The fit function, $F(R)=A \exp (-B R)$ with $A=1.505$ and $B=1.347 \mathrm{fm}^{-1}$, is shown as a solid line.

$\beta=5.7,5.8$, and 6.0 so that one can see the $\beta$ (lattice spacing) dependence. While a tiny deviation is found among three $\beta$ 's, all the data seem lie in a monotonous line, which means the systematic errors for $A$ and $B$ mainly arise from the lattice size $L$. For $L>5 \mathrm{fm}$, the fitted values are stable, and $A$ and $B$ are determined as

$$
\begin{gathered}
A=1.505(49) \\
B=1.347(35) \mathrm{fm}^{-1}=265(7) \mathrm{MeV}
\end{gathered}
$$

from $F(R)$ obtained in the largest volume.

In Fig. 8, the singlet component $\rho(R)_{\mathbf{1}, \mathbf{1}}$ and the averaged octet component $\rho(R)_{8,8}$ are plotted as a function of the interquark distance $R$. One finds that both components approach $\rho(R)_{\mathbf{1 , 1}}=\rho(R)_{\mathbf{8}, \mathbf{8}}=\frac{1}{N_{c}^{2}}=\frac{1}{9}$ at large $R$, which ensures that the reduced density matrix at large interquark separation $R$ is governed by the random component $\hat{\rho}^{\text {rand }}$ and the original correlated state $\hat{\rho}^{0}$ vanishes.

\section{Finite volume effects}

Within the present numerical accuracy, the only independent quantity in the reduced density matrix $\rho(R)$ is $\rho_{\mathbf{8 , \boldsymbol { 8 }}}$,
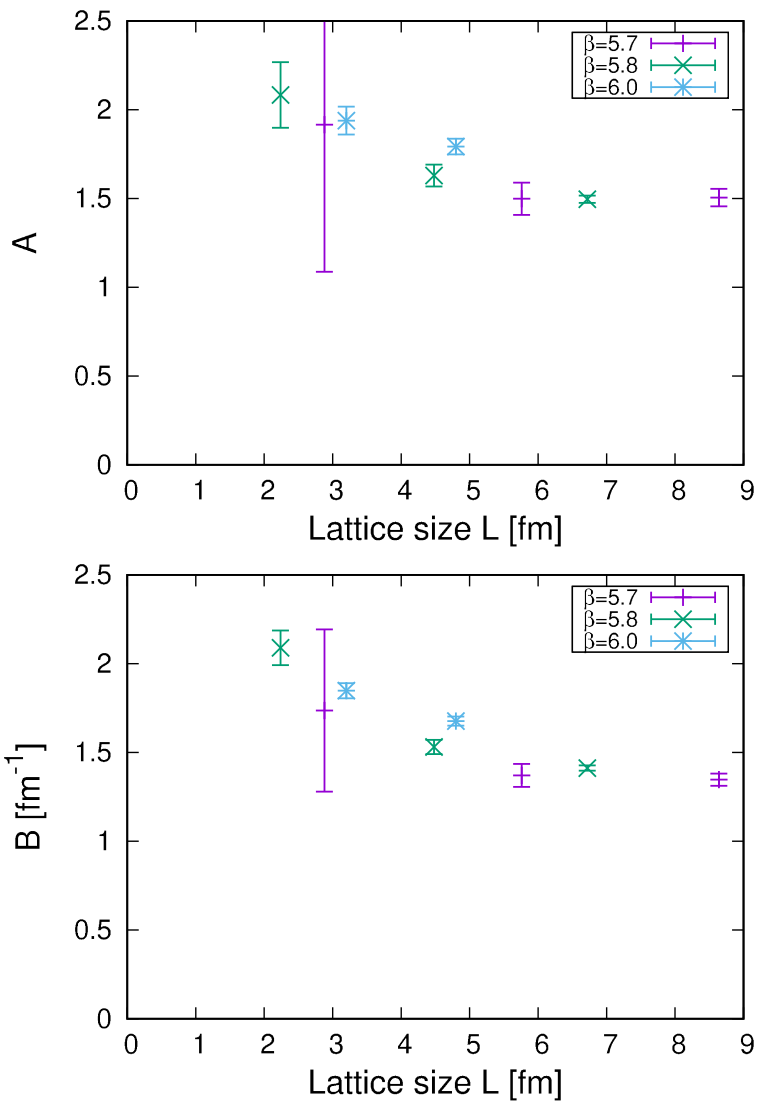

FIG. 7. The fitted parameters $A$ and $B$ plotted as functions of the spatial lattice size $L$.

and all the finite volume effects are reflected in $F(R)=$ $1-N_{c}^{2} \rho(R)_{\mathbf{8 . 8}}$.

In Fig. 9, $F(R)$ for several $L$ (lattice size) and $\beta$ (lattice spacing) are plotted as a function of the interquark distance $R$. At $L>5.0 \mathrm{fm}, F(R)$ shows almost no volume dependence, and $\rho(R)$ is safe from the finite volume effects at this $L$ range. When the lattice size $L$ is small, $F(R)$ rapidly decreases with increasing $R$. On the other hand, $\beta$ dependence seems smaller than the finite volume effect. The systematic errors mainly come from the finite size effect.

This finite volume effect would be due to the periodic boundary condition, with which identical $q \bar{q}$ systems exist with the period $L$. Quark and antiquark $[q(0) \bar{q}(R)]$ separated by $R$ in a system can also form color-singlet pairs with quarks that are separated with the distance $L-R$, which additionally enters in $\rho(R)$ as a random mixture decreasing $F(R)$.

\section{E. Entanglement entropy}

In the following, we consider the $\alpha=2$ case for the evaluation of the EE. (We will go back to $S^{\mathrm{VN}}$ in the latter part of this section.) The $S^{\text {Renyi-2 }}(R)$ is correctly calculated from the trace of the squared reduced density matrix $\rho(R)$ as

$$
S^{\text {Renyi-2 }}=-\log \operatorname{Tr}\left(\rho(R)^{2}\right) .
$$

Taking into account that $\operatorname{Tr}(\rho(R))=1$, the maximum of $S^{\text {Renyi-2 }}$ is obtained when all the $N_{c}^{2}$ diagonal elements are 


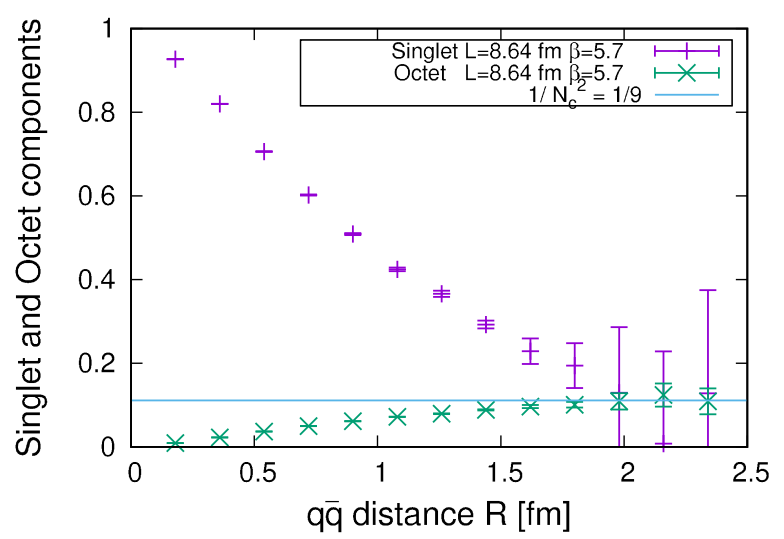

FIG. 8. The singlet component $\rho(R)_{1,1}$ and the averaged octet component $\rho(R)_{\mathbf{8 , 8}}$ are plotted as a function of the interquark distance $R$. Both are approaching $1 / N c^{2}=1 / 9$ at large $R$.

equal to $1 / N_{c}^{2}$ in the diagonal representation of $\rho(R)$. From the representation invariance of $S$, the maximum value of $S$ is proved to be

$$
\max \left[S^{\text {Renyi-2 }}(R)\right]=2 \log N_{c} .
$$

In Fig. 10, $S^{\text {Renyi-2 }}(R)$ calculated with the $\rho(R)$ obtained on the lattice are plotted as $S_{\text {lattice }}^{\text {Renyi-2 }}(R) . S_{\text {lattice }}^{\text {Renyi-2 }}(R)$ approaches $2 \log N_{c}$ as $R$ increases, which indicates that $\rho(R)$ is described by the random component $\hat{\rho}^{\text {rand }}$ in the large $R$ limit.

In the ansatz, the density matrix $\rho_{\text {ansatz }}(R)$ is a diagonal matrix, and $\operatorname{Tr}\left(\rho_{\text {ansatz }}(R)^{2}\right)$ is given by $F(R)$ as

$$
\operatorname{Tr}\left(\rho_{\text {ansatz }}(R)^{2}\right)=F(R)^{2}+\frac{1}{N_{c}^{2}}-\frac{F(R)^{2}}{N_{c}^{2}}
$$

Then, $S_{\text {ansatz }}^{\text {Reni-2 }}(R)$, the Renyi entropy evaluated using the ansatz, is expressed as

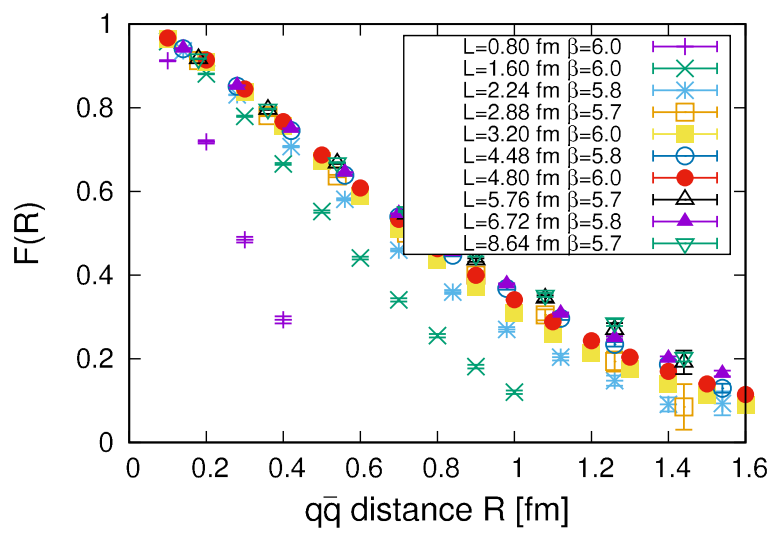

FIG. 9. $F(R)$ for different $L$ (lattice size) are plotted as a function of the interquark distance $R$. At $L>5.0 \mathrm{fm}, F(R)$ shows almost no volume dependence, and $\rho(R)$ is safe from the finite volume effects at this $L$ range.

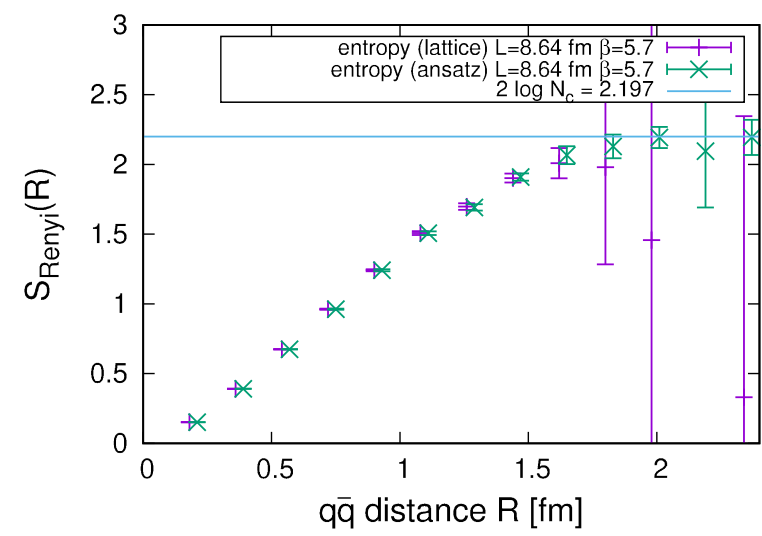

FIG. 10. $\quad S_{\text {lattice }}^{\text {Renyi-2 }}(R)$ obtained from the original reduced density matrix $\rho(R)$ and $S_{\text {ansatz }}^{\text {Renyi-2 }}(R)$ obtained using the ansatz are plotted as a function of the interquark distance $R$.

$$
\begin{aligned}
S_{\text {ansatz }}^{\text {Renyi-2 }}(R) & =-\log \operatorname{Tr}\left(\rho_{\text {ansatz }}(R)^{2}\right) \\
& =-\log \left(F(R)^{2}+\frac{1}{N_{c}^{2}}-\frac{F(R)^{2}}{N_{c}^{2}}\right) .
\end{aligned}
$$

Figure 10 shows $S_{\text {ansatz }}^{\text {Renyi-2 }}(R)$ obtained using the ansatz plotted as a function of the interquark distance $R$. $S_{\text {ansatz }}^{\text {Renyi-2 }}(R)$ approaches $2 \log N_{c}$ at large $R$, which again confirms that $F(R)$ goes to zero and $\rho_{\text {ansatz }}(R)$ is expressed by the random elements $\hat{\rho}^{\text {rand }}$ in the $R \rightarrow \infty$ limit. The remarkable fact is that $S_{\text {lattice }}^{\text {Reni-2 }}(R)$ and $S_{\text {ansatz }}^{\text {Renyi-2 }}(R)$ are almost identical for all $R$, which indicates that the reduced density matrix $\rho(R)$ can be expressed very well by the ansatz.

$S_{\text {ansatz }}^{\text {Renyi-2 }}$ and $S_{\text {lattice }}^{\text {Renyi-2 }}$ for different lattice sizes $L$ are plotted as a function of $R$ in Fig. 11. As expected, when $L<5.0 \mathrm{fm}$, the finite volume effects are rather large, and $S_{\text {ansatz }}^{\text {Reni-2 }}$ and $S_{\text {lattice }}^{\text {Renyi-2 }}$ are both affected. On the other hand, for all the $L$, $S_{\text {ansatz }}^{\text {Reni-2 }} \simeq S_{\text {lattice }}^{\text {Reni-2 }}$ is found, and the ansatz is valid with good accuracy even when the finite volume effects are large.

It is well known that any averaging leads to the growth of the entropy. The reduced density-matrix components are averaged in the ansatz, and one may think $S_{\text {ansatz }}^{\text {Renyi-2 }}>$ $S_{\text {lattice }}^{\text {Reni-2 }}$ should be observed. Although such a tendency can be sometimes seen in figures, statistical errors are much larger, and both data are consistent with each other within the present statistics.

Finally, we show the von Neumann entropy $S^{\mathrm{VN}}$ based on the ansatz. The direct calculation of $S^{\mathrm{VN}}$ from the reduced density matrix on the lattice is numerically demanding. Instead of such a straightforward approach, we take an alternative way to calculate $S^{\mathrm{VN}}$ with an approximation using $\rho_{\text {ansatz }}$ from the ansatz as

$$
S_{\text {ansatz }}^{\mathrm{VN}}=-\operatorname{Tr}\left(\rho_{\text {ansatz }} \log \rho_{\text {ansatz }}\right) .
$$

$\rho$ evaluated on the lattice coincides with $\rho_{\text {ansatz }}$ with high accuracy as shown above, and $S_{\text {ansatz }}^{\mathrm{VN}}$ is expected to be a good approximation of $S^{\mathrm{VN}}$. Now, the reduced density 


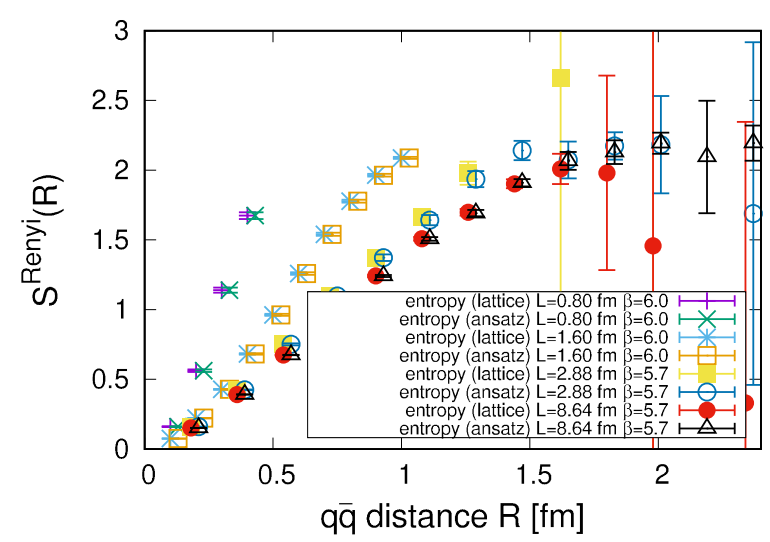

FIG. 11. $S_{\text {ansatz }}^{\text {Renyi-2 }}$ and $S_{\text {lattice }}^{\text {Renyi-2 }}$, which are obtained from the original reduced density matrix $\rho(R)$ and that obtained using the ansatz, are plotted as a function of $R$.

matrix in the $\alpha$ representation has been found to be diagonal, and then $S^{\mathrm{VN}}(R)$ is easily computed as

$$
\begin{aligned}
S_{\mathrm{ansatz}}^{\mathrm{VN}}(R)= & -\left(F(R)+\frac{1}{N_{c}^{2}}(1-F(R))\right) \\
& \times \log \left(F(R)+\frac{1}{N_{c}^{2}}(1-F(R))\right) \\
& -\left(N_{c}^{2}-1\right)\left(\frac{1}{N_{c}^{2}}(1-F(R))\right) \\
& \times \log \left(\frac{1}{N_{c}^{2}}(1-F(R))\right) .
\end{aligned}
$$

Figure 12 shows $S_{\text {ansatz }}^{\mathrm{VN}}(R)$ as a function of $R$, and $S_{\text {lattice }}^{\text {Renyi-2 }}(R)$ and $S_{\text {ansatz }}^{\text {Renyi-2 }}(R)$ are also plotted for reference. $S_{\text {ansatz }}^{\mathrm{VN}}(R)$ increases toward $2 \log N_{c}$ faster than $S^{\text {Renyi-2}}(R)$ as the VN $\mathrm{EE}$ is a more sensitive measure of the entanglement than the Renyi-2 EE in general. As $R$ increases, the reduced density matrix $\hat{\rho}$ is dominated by the random contribution $\hat{\rho}^{\text {rand }}$, and all the matrix elements are equipartitioned in this limit giving the maximum value of entropy.

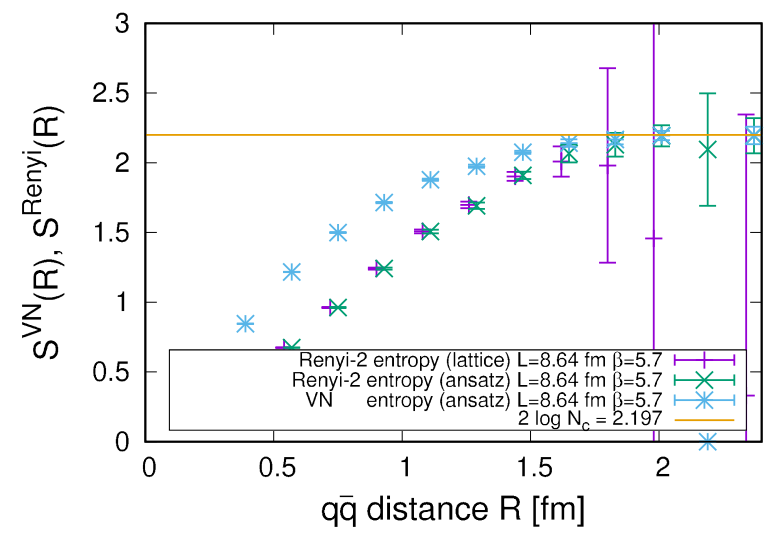

FIG. 12. $S_{\text {ansatz }}^{\mathrm{VN}}, S_{\text {ansatz }}^{\text {Renyi-2 }}$, and $S_{\text {lattice }}^{\text {Renyi-2 }}$, which are obtained from the original reduced density matrix $\rho(R)$ and that obtained using the ansatz, are plotted as a function of $R$.

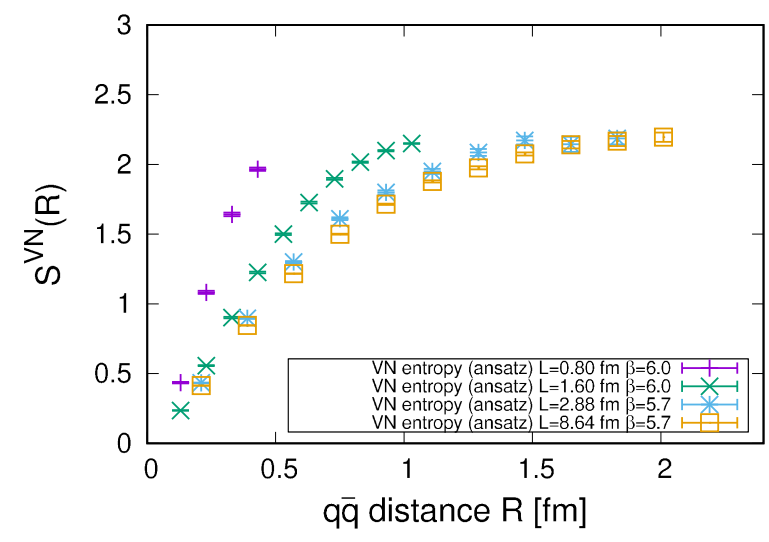

FIG. 13. $S_{\text {ansatz }}^{\mathrm{VN}}$ obtained using the ansatz are plotted as a function of $R$ for different lattice size $L$.

To see the finite volume effects, we plot $S_{\text {ansatz }}^{\mathrm{VN}}$ as a function of $R$ in Fig. 13. The tendency that $S$ is increased by the finite size effects remains unchanged.

\section{CONCLUSIONS}

We have studied the color correlation of static quark and antiquark $(q \bar{q})$ systems in the confined phase from the viewpoint of the entanglement entropy defined by reduced density matrices $\rho$ in color space. We have adopted the standard Wilson gauge action and performed quenched calculations for density matrices. The gauge couplings are $\beta=5.7,5.8$, and 6.0, and the spatial volumes are $L^{3}=8^{3}$, $16^{3}, 32^{3}$, and $48^{3}$. To evaluate each component of $\rho_{i j, k l}$, all the gauge configurations are Coulomb-gauge fixed. We have also proposed an ansatz for the reduced density matrix $\rho$, in which $\rho$ is written by a sum of the color-singlet (correlated) state $|\mathbf{1}\rangle\langle\mathbf{1}|$ and random (uncorrelated) elements $|\mathbf{1}\rangle\langle\mathbf{1}|$, $\left|\mathbf{8}_{i}\right\rangle\left\langle\mathbf{8}_{i}\right|\left(i=1, \ldots, N_{c}^{2}-1\right)$ induced by the QCD interaction.

We have quantitatively evaluated the $q \bar{q}$ correlation by means of the entanglement entropy constructed from the reduced density matrix $\rho$. We have adopted the von Neumann entropy $S^{\mathrm{VN}}$ and the Renyi entropy of the order $\alpha S^{\text {Renyi- } \alpha}$ for the evaluation of EE. Especially when $\alpha$ is an integer, $S^{\text {Renyi- } \alpha}$ can be computed easily from the density matrix product $\rho^{\alpha}$, and we need no diagonalization of $\rho$. Note that color indices in EEs are all contracted, and colorcorrelation measurement by means of EEs can be performed in a gauge- (representation-)independent way.

As a result, we have found that the reduced density matrix $\rho$ can be reproduced well with the ansatz: The reduced density matrix $\rho$ consists of the color-singlet (correlated) state $|\mathbf{1}\rangle\langle\mathbf{1}|$ when the $q \bar{q}$ distance is small, and random (uncorrelated) diagonal elements $|\mathbf{1}\rangle\left\langle\mathbf{1}|,| \mathbf{8}_{i}\right\rangle\left\langle\mathbf{8}_{i}\right|(i=1, \ldots$, $\left.N_{c}^{2}-1\right)$ are equally mixed as the $q \bar{q}$ distance is increased. The $q \bar{q}$ color correlations have been found to be quantified well by entanglement entropies, and we conclude that entanglement entropy can be a gauge-independent measure for color correlations. 


\section{ACKNOWLEDGMENTS}

This work was partly supported by Grants-in-Aid of the Japan Society for the Promotion of Science (Grants No. 16K05365, No. JP18K03617, and No. 18H05407).

\section{APPENDIX: $\alpha$ REPRESENTATION AND $i j$ REPRESENTATION}

In SU(3) QCD, the $q \bar{q}$ state in $\alpha$ representation, $|\mathbf{1}\rangle$ and $\left|\mathbf{8}_{i}\right\rangle\left(i=1,2, \ldots, N_{c}^{2}-1\right)$, can be expressed by states in $i j$ representation, $\left|\bar{q}_{i} q_{j}\right\rangle$, as follows:

$$
\begin{aligned}
|\mathbf{1}\rangle & =\frac{1}{\sqrt{3}}\left(\sum_{i}\left|\bar{q}_{i} q_{i}\right\rangle\right), \\
\left|\mathbf{8}_{1}\right\rangle & =-\left|\bar{q}_{1} q_{2}\right\rangle, \\
\left|\mathbf{8}_{2}\right\rangle & =-\frac{1}{\sqrt{2}}\left(\left|\bar{q}_{1} q_{1}\right\rangle-\left|\bar{q}_{2} q_{2}\right\rangle\right), \\
\left|\mathbf{8}_{3}\right\rangle & =\left|\bar{q}_{2} q_{1}\right\rangle, \\
\left|\mathbf{8}_{4}\right\rangle & =\left|\bar{q}_{1} q_{3}\right\rangle, \\
\left|\mathbf{8}_{5}\right\rangle & =-\left|\bar{q}_{2} q_{3}\right\rangle, \\
\left|\mathbf{8}_{6}\right\rangle & =\left|\bar{q}_{3} q_{2}\right\rangle, \\
\left|\mathbf{8}_{7}\right\rangle & =\left|\bar{q}_{3} q_{1}\right\rangle, \\
\left|\mathbf{8}_{8}\right\rangle & =\frac{1}{\sqrt{6}}\left(\left|\bar{q}_{1} q_{1}\right\rangle+\left|\bar{q}_{2} q_{2}\right\rangle-2\left|\bar{q}_{3} q_{3}\right\rangle\right) .
\end{aligned}
$$

Then, the elements of $\hat{\rho}$ in $\alpha$ represenation can be related with those in $i j$ representation as

$$
\begin{aligned}
\hat{\rho}_{\mathbf{1}, \mathbf{1}}= & |\mathbf{1}\rangle\langle\mathbf{1}| \\
= & \frac{1}{3}\left(\sum_{i}\left|\bar{q}_{i} q_{i}\right\rangle\right)\left(\sum_{i}\left\langle\bar{q}_{i} q_{i}\right|\right) \\
= & \frac{1}{3} \sum_{i j} \hat{\rho}_{i i, j j}, \\
\hat{\rho}_{\mathbf{8}_{1}, \mathbf{8}_{1}}= & \left|\mathbf{8}_{1}\right\rangle\left\langle\mathbf{8}_{1}|=| \bar{q}_{1} q_{2}\right\rangle\left\langle\bar{q}_{1} q_{2}\right|=\hat{\rho}_{12,12}, \\
\hat{\rho}_{\mathbf{8}_{2}, \mathbf{8}_{2}}= & \left|\mathbf{8}_{2}\right\rangle\left\langle\mathbf{8}_{2}\right|=\frac{1}{2}\left(\hat{\rho}_{11,11}+\hat{\rho}_{22,22}-\hat{\rho}_{11,22}-\hat{\rho}_{22,11}\right), \\
\hat{\rho}_{\mathbf{8}_{3}, \mathbf{8}_{3}}= & \left|\mathbf{8}_{3}\right\rangle\left\langle\mathbf{8}_{3}|=| \bar{q}_{2} q_{1}\right\rangle\left\langle\bar{q}_{2} q_{1}\right|=\hat{\rho}_{21,21}, \\
\hat{\rho}_{\mathbf{8}_{4}, \mathbf{8}_{4}}= & \left|\mathbf{8}_{4}\right\rangle\left\langle\mathbf{8}_{4}|=| \bar{q}_{1} q_{3}\right\rangle\left\langle\bar{q}_{1} q_{3}\right|=\hat{\rho}_{13,13}, \\
\hat{\rho}_{\mathbf{8}_{5}, \mathbf{8}_{5}}= & \left|\mathbf{8}_{5}\right\rangle\left\langle\mathbf{8}_{5}|=| \bar{q}_{2} q_{3}\right\rangle\left\langle\bar{q}_{2} q_{3}\right|=\hat{\rho}_{23,23}, \\
\hat{\rho}_{\mathbf{8}_{6}, \mathbf{8}_{6}}= & \left|\mathbf{8}_{6}\right\rangle\left\langle\mathbf{8}_{6}|=| \bar{q}_{3} q_{2}\right\rangle\left\langle\bar{q}_{3} q_{2}\right|=\hat{\rho}_{32,32}, \\
\hat{\rho}_{\mathbf{8}_{7}, \mathbf{8}_{7}}= & \left|\mathbf{8}_{7}\right\rangle\left\langle\mathbf{8}_{7}|=| \bar{q}_{3} q_{1}\right\rangle\left\langle\bar{q}_{3} q_{1}\right|=\hat{\rho}_{31,31}, \\
\hat{\rho}_{\mathbf{8}_{8}, \mathbf{8}_{8}}= & \left|\mathbf{8}_{8}\right\rangle\left\langle\mathbf{8}_{8}\right| \\
= & \frac{1}{6}\left(\hat{\rho}_{11,11}+\hat{\rho}_{11,22}+\hat{\rho}_{22,11}+\hat{\rho}_{22,22}\right. \\
& \left.-2 \hat{\rho}_{11,33}-2 \hat{\rho}_{22,33}-2 \hat{\rho}_{33,11}-2 \hat{\rho}_{33,22}+4 \hat{\rho}_{33,33}\right) .
\end{aligned}
$$

[1] J. Greensite, An Introduction to the Confinement Problem, Lecture Notes in Physics (Springer Heidelberg, Germany, 2011), and references therein.

[2] G. S. Bali, K. Schilling, and C. Schlichter, Phys. Rev. D 51, 5165 (1995).

[3] V. G. Bornyakov, H. Ichie, Y. Mori, D. Pleiter, M. I. Polikarpov, G. Schierholz, T. Streuer, H. Stüben, and T. Suzuki (DIK Collaboration), Phys. Rev. D 70, 054506 (2004).

[4] G. Tiktopoulos, Phys. Lett. 66B, 271 (1977).

[5] J. Greensite and C. B. Thorn, J. High Energy Phys. 02 (2002) 014.

[6] E. Itou, K. Nagata, Y. Nakagawa, A. Nakamura, and V. I. Zakharov, Prog. Theor. Exp. Phys. 2016, 061 B01 (2016).

[7] S. Aoki, T. Iritani, M. Nozaki, T. Numasawa, N. Shiba, and H. Tasaki, J. High Energy Phys. 06 (2015) 187.

[8] Y. Kanada-En'yo, Phys. Rev. C 91, 034303 (2015).
[9] T. Takayanagi, Classical Quantum Gravity 29, 153001 (2012).

[10] C. H. Bennett, H. J. Bernstein, S. Popescu, and B. Schumacher, Phys. Rev. A 53, 2046 (1996).

[11] P. Calabrese and J. L. Cardy, J. Stat. Mech. (2005) P06002.

[12] G. Vidal, J. I. Latorre, E. Rico, and A. Kitaev, Phys. Rev. Lett. 90, 227902 (2003).

[13] L. Amico, R. Fazio, A. Osterloh, and V. Vedral, Rev. Mod. Phys. 80, 517 (2008).

[14] R. Horodecki, P. Horodecki, M. Horodecki, and K. Horodecki, Rev. Mod. Phys. 81, 865 (2009).

[15] C. H. Bennett, D. P. DiVincenzo, J. A. Smolin, and W. K. Wootters, Phys. Rev. A 54, 3824 (1996).

[16] W. K. Wootters, Phys. Rev. Lett. 80, 2245 (1998).

[17] G. Vidal and R.F. Werner, Phys. Rev. A 65, 032314 (2002).

[18] A. Renyi, Probability Theory (Elsevier Science Publishing Co Inc., New York, USA, 1970). 